九州大学学術情報リポジトリ

Kyushu University Institutional Repository

\title{
AN OPTIMALITY EQUATION IN CONTROLLED JUMP PROCESSES WITH A CHOICE OF STOPPING RULES
}

Otsubo, Yoshio

Department of Mathematics, Faculty of science, Kyushu University

https://doi.org/10.5109/13138

出版情報: 統計数理研究. 19 (1/2)，pp. 35-44，1980-03. Research Association of Statistical Sciences

バージョン :

権利関係 : 


\title{
AN OPTIMALITY EQUATION IN CONTROLLED JUMP PROCESSES WITH A CHOICE OF STOPPING RULES
}

\author{
By
}

\author{
Yoshio Oнтsubo*
}

(Received September 10, 1979)

\section{Introduction}

Controlled Markov jump processes associated with a choice of stopping rules on general state and action spaces have been first introduced by Ohtsubo [5]. In that paper the author has given the optimal return in the form of the limit of successive approximations and also given necessary and sufficient conditions for a control (a pair of policy and stopping rule) to be optimal in terms of an inequality and a functional equation involving an infinitesimal operator. The latter result, however, is valid only when an optimal control exists. In general, the functional equation arising in the optimization of controlled processes is the so-called optimality equation that should be satisfied by an optimal return over infinite future. Main purpose of the present paper is to make a study of the functional equation in connection with the optimal return in controlled jump processes with a choice of stopping rules.

Kakumanu [4] and Doshi [2] have shown that the optimal return in a certain type of continuous time Markov decision model satisfies the optimality equation. Shiryayev [6] and Furukawa [3] have studyed optimality equations in optimal stopping problems and in stopped decision problems, respectively.

In Section 2, we shall introduce notations and definitions to be used throughout this paper, and in Section 3 we shall set up several assumptions and prepare fundamental lemmas to be used in Section 4. In Section 4, we shall show that the optimal return satisfies a functional equation involving an infinitesimal operator, that is to say, an optimality equation.

\section{Preliminaries}

The state space $S$ is a non-empty Borel subset of a complete separable metric space. $Z$ is the cartesian product of $S$ and $R^{+}$where the time space $R^{+}$is the set of all non-negative real numbers. The action space $A$ is a non-empty Borel subset of a complete separable metric space. $\mathscr{B}(S), \mathscr{B}(Z)$ and $\mathscr{B}(A)$ mean $\sigma$-fields of Borel subsets of $S, Z$ and $A$, respectively. The terminal reward function $g$ is a bounded

\footnotetext{
* Department of Mathematics, Faculty of Science, Kyushu University, Fukuoka.
} 
function on $Z$ such that for each $t \geqq 0 g(\cdot, t)$ is $\mathcal{B}(S)$-measurable, and for each $x \in S$ $g(x, \cdot)$ is right continuous on $R^{+}$.

A mapping $\pi$ from $Z$ to $A$ is called an admissible policy if for each $t \geqq 0 \pi(\cdot, t)$ is $\mathscr{B}(S) / \mathscr{B}(A)$-measurable and for each $x \in S \pi(x, \cdot)$ is piecewise constant, right continuous on $R^{+}$and has only a finite number of discontinuities on any finite interval. Let $\Pi$ denote the set of all admissible policies.

In order to determine the processes, we need to introduce a real-valued function $\lambda$ on $Z \times A$ and a Markov kernel $Q$. We assume the following conditions:

(i) For each $t \geqq 0, \lambda(\cdot, t, \cdot)$ is $\mathscr{B}(S) \times \mathscr{B}(A)$-measurable.

(ii) For each $(x, a) \in S \times A, \lambda(x, \cdot, a)$ is right continuous on $R^{+}$.

(iii) There exists a natural number $M<\infty$ such that $0<\lambda(z, a)<M$ for all $(z, a)$ $\in Z \times A$.

(iv) For each $(z, a) \in Z \times A, Q(\cdot \mid z, a)$ is a probability measure on $\mathscr{B}(S)$.

(v) For each $(x, t, a) \in Z \times A, Q(\{x\} \mid x, t, a)=0$.

(vi) For each $(t, B) \in R^{+} \times \mathscr{B}(S), Q(B \mid \cdot, t, \cdot)$ is $\mathscr{B}(S) \times \mathscr{B}(A)$-measurable.

(vii) For each $(x, a) \in S \times A, Q(B \mid x, \cdot, a)$ is piecewise constant, right continuous on $R^{+}$and has only a finite number of discontinuities on any finite interval, independently of all $B \in \mathscr{B}(S)$.

The jump process $Z^{\pi}=\left(Z_{t}^{\pi}\right)_{t \geq 0}=\left(X_{t}^{\pi}, t\right)_{t \geqq 0}$ (corresponding to $\pi \in \Pi$ ) is defined by the following probability laws:

$$
\begin{aligned}
& P_{(x, s)}^{\pi}[\nu(x, s) \leqq t]=1-\exp \left\{-\int_{s}^{s+t} \lambda\left(x, s^{\prime}, \pi\left(x, s^{\prime}\right)\right) d s^{\prime}\right\}, \\
& P_{(x, s)}^{\pi}\left[X_{s+t}^{\pi} \in B \mid \nu(x, s)=t\right]=Q(B \mid x, s+t, \pi(x, s+t))
\end{aligned}
$$

for each $(x, s, t) \in Z \times R^{+}$and any $B \in \mathcal{B}(S)$, where $\nu(x, s)$ is a holding time such that the $x$-component of the process starts in $x$ at time $s$ and remains there a random time $\nu(x, s)$. Then the process $Z^{\pi}$ is a Markov jump process.

By Blumental and Getoor [1] we may regard the sample space $\Omega$ as the set of all functions $\omega: R^{+} \rightarrow Z$ such that (i) for each $t \geqq 0$ there exists a state $y \in S$ satisfying $\omega(t)=(y, t)$, (ii) for each $t \geqq 0$ a left-hand limit $\omega(t-0)$ does exist, and (iii) for each $t \geqq 0$ if $\omega(t)=(x, t) \in Z$ then there exists a $h>0$ such that $\omega\left(t+h^{\prime}\right)=\left(x, t+h^{\prime}\right)$ for all $h^{\prime} \in[0, h)$.

For each $0 \leqq s \leqq t$ let $\mathscr{F}_{t}^{s}$ denote the $\sigma$-field of subsets of $\Omega$ generated by the sets $\left\{\omega \in \Omega \mid \omega\left(s^{\prime}\right) \in B\right\}, B \in \mathscr{B}(Z), s \leqq s^{\prime} \leqq t$.

For each $\pi \in \Pi$ and each $s \geqq 0$ let $C_{s}(\pi)$ denote the set of all stopping times $\tau$ with respect to $\left\{\mathscr{F}_{t}^{s}\right\}_{t \geq s}$ satisfying that $P_{(x, s)}^{\pi}(s \leqq \tau<\infty)=1$ for all $x \in S$.

The expected terminal return $\varphi_{\tau}^{\pi}$ of our system corresponding to $\pi \in \Pi$ and $\tau \in$ $C_{s}(\pi)$ is defined by

$$
\varphi_{\tau}^{\pi}(x, s)=E_{(x, s ;}^{\pi}\left[g\left(Z_{\tau}^{\pi}\right)\right] \quad \text { for }(x, s) \in Z,
$$

where $E_{(x, s)}^{\pi}$ denotes the expectation operator with respect to $P_{(x, s)}^{\pi}$.

The optimal return $u^{*}$ is defined by

$$
u^{*}(x, s)=\sup _{\pi \in \Pi, \tau \in C_{s}(\pi)} \varphi_{\tau}^{\pi}(x, s) \quad \text { for } \quad(x, s) \in Z .
$$


DEFinition 2.1. Let $f$ be a bounded universally measurable function on $Z$.

(i) $f$ is said to be excessive, if it holds that for each $(x, s) \in Z$ and any $\pi \in I I$

and

$$
f(x, s) \geqq E_{(x, s)}^{\pi}\left[f\left(Z_{t}^{\pi}\right)\right] \quad \text { for all } t \geqq s,
$$

$$
\lim _{t \downarrow s} \inf f\left(Z_{t}^{\pi}\right) \geqq f(x, s) \quad P_{(x, s)}^{\pi}-\text { a.s. . }
$$

(ii) $f$ is called a majorant of $g$, if $f(z) \geqq g(z)$ for all $z \in Z$.

(iii) An excessive majorant $f$ of $g$ is called the smallest excessive majorant of $g$, if for any excessive majorant $h$ of $g, f(z) \leqq h(z)$ for all $z \in Z$.

Let $F$ be the set of all bounded Borel measurable functions on $Z$. For each $\pi \in \Pi$ let $\tilde{F}^{\bar{x}}$ be the set of all $f \in F$ such that

$$
\lim _{t \downarrow 0} E_{(x, s)}^{\pi}\left[f\left(Z_{s+t}^{\pi}\right)\right]=f(x, s) \quad \text { for } \quad(x, s) \in Z .
$$

The weak infinitesimal operator (corresponding to $\pi \in \Pi$ ) is defined by

$$
\mathcal{A}^{\pi} f(x, s)=\lim _{t \downarrow 0} \frac{1}{t}\left\{E_{(x, s)}^{\pi}\left[f\left(Z_{s+t}^{\pi}\right)\right]-f(x, s)\right\}, \quad(x, s) \subseteq Z,
$$

for all $f \in \tilde{F}^{-}$such that the limit of the right-hand side exists in the weak sence and belongs to $\widetilde{F}^{\pi}$. Let $\mathscr{D}\left(\mathcal{A}^{\pi}\right) \subset \widetilde{F}^{\pi}$ denote the set of such functions and let $\mathscr{D}(\mathcal{A})$ $=\bigcap_{\pi \in I I} \mathscr{D}\left(\mathscr{A}^{\pi}\right)$.

In the remainder of this paper, even in the case when a random variable $\hat{\tau}$ is defined depending on a policy $\pi$ and a time $s$, we shall not use an explicit expression such as $\hat{\tau}(\pi, s)$ but use the simple notation $\hat{\tau}$ (or, if we need to more precisely, we use the notation $\hat{\tau}(s)$ ).

\section{Fundamental lemmas}

We have proved in the previous paper [5] that the optimal return $u^{*}$ is a universally measurable function on $Z$ and the smallest excessive majorant of $g$, and that for each $x \in S u^{*}(x, \cdot)$ is right continuous on $R^{+}$. In this section, by using these results, some fundamental lemmas to be used in the following section will be prepared.

Let

$$
\Gamma^{*}=\left\{z \in Z \mid u^{*}(z)=g(z)\right\} .
$$

For each integer $m \geqq 1$, each $s \geqq 0$ and any $\pi \in \Pi$, let

and

$$
\begin{aligned}
& \Gamma_{m}^{*}=\left\{z \in Z \mid u^{*}(z) \leqq g(z)+\frac{1}{m}\right\}, \\
& \tau_{m}=\tau_{m}(s)=\inf \left\{t>s \mid Z_{t}^{\pi} \in \Gamma_{m}^{*}\right\}, \\
& \sigma_{m}=\inf \left\{t \geqq s \mid Z_{t}^{\pi} \in \Gamma_{m}^{*}\right\} .
\end{aligned}
$$

Then it is obvious that $\Gamma^{*}=\bigcap_{m=1}^{\infty} \Gamma_{m}^{*}$ and $\Gamma_{m}^{*} \supset \Gamma_{n}^{*}$ for each $n \geqq m$. For each $m \geqq 1$, let's define

$$
u_{m}(\pi)(z)=E_{z}^{\pi}\left[u^{*}\left(Z_{\tau_{m}}^{\pi}\right)\right] \quad \text { for } \quad z \in Z \text { and } \pi \in \Pi \text {, }
$$


and

$$
\tilde{u}_{m}(z)=\sup _{\pi \in I I} u_{m}(\pi)(z) \quad \text { for } \quad z \in Z .
$$

Throughout this paper we impose the following assumptions.

Assumption (A). (i) For each integer $m \geqq 1, \tilde{u}_{m}$ is universally measurable on $Z$.

(ii) Let $m$ be any natural number and let $p$ be any probability measure on $\mathcal{B}(S)$. Then for given $\varepsilon>0$ and $t \geqq 0$, there exists a policy $\pi_{\varepsilon, t} \in \Pi$ such that

$$
p\left\{x \in S: u_{m}\left(\pi_{\varepsilon, t}\right)(x, t)+\varepsilon \geq \tilde{u}_{m}(x, t)\right\}=1 .
$$

Assumption (B). For any $f \subseteq \mathscr{D}(\mathcal{A})$ and for each $\varepsilon>0$, there exists a policy $\pi_{\varepsilon} \in$ $\Pi$ such that

$$
\sup _{\pi \in I I} \mathcal{A}^{\pi} f(z)<\mathcal{A}^{\pi} \varepsilon f(z)+\varepsilon \quad \text { for all } z \notin \Gamma^{*} .
$$

LEMMA 3.1. For each $m \geqq 1, \tilde{u}_{m}$ is excessive.

Proof. Let $m \geqq 1$ be fixed but arbitrary.

First we shall show that for each $z=(x, s) \in Z$ and any $\pi \in I$,

$$
\tilde{u}_{m}(z) \geqq E_{z}^{\pi}\left[\tilde{u}_{m}\left(Z_{t}^{\pi}\right)\right] \quad \text { for all } t \geqq s .
$$

Let $z=(x, s) \in Z, \pi \in \Pi$ and $t \geqq s$ be arbitrary. From Assumption (A) (ii), it holds that for any $\varepsilon>0$ there exists a policy $\pi_{s, t} \in \Pi$ such that

Thus we have

$$
P_{2}^{\pi}\left[u_{m}\left(\pi_{s, t}\right)\left(Z_{t}^{\pi}\right)+\varepsilon \geqq \tilde{u}_{m}\left(Z_{t}^{\pi}\right)\right]=1 .
$$

$$
E_{z}^{\pi}\left[\tilde{u}_{m}\left(Z_{t}^{\pi}\right)\right] \leqq E_{z}^{\pi}\left[u_{m}\left(\pi_{s, t}\right)\left(Z_{t}^{\pi}\right)\right]+\varepsilon .
$$

We define a policy $\hat{\kappa}$ by

$$
\hat{\pi}\left(y, t^{\prime}\right)= \begin{cases}\pi\left(y, t^{\prime}\right) & \text { if } t^{\prime}<t, \\ \pi_{s, t}\left(y, t^{\prime}\right) & \text { if } t^{\prime} \geqq t .\end{cases}
$$

Then $\hat{\pi} \in I I$. Since $u^{*}$ is excessive and $\tau_{m}(s) \leqq \tau_{m}(t)$,

$$
\begin{aligned}
E_{z}^{\pi}\left[u_{m}\left(\pi_{s, t}\right)\left(Z_{t}^{\pi}\right)\right] & =E_{z}^{\pi}\left[E_{z_{t}^{\pi}}^{\pi_{\varepsilon_{t}, t}^{\pi}}\left[u^{*}\left(Z_{\tau_{m}}^{\pi_{s}, t}\right)\right]\right] \\
& =E_{z}^{\hat{\pi}}\left[u^{*}\left(Z_{\tau_{m}(t)}^{\hat{\pi}}\right)\right] \\
& \leqq E_{z}^{\hat{\pi}}\left[u^{*}\left(Z_{\tau_{m}(s)}^{\hat{\pi}}\right)\right] \\
& \leqq \tilde{u}_{m}(z) .
\end{aligned}
$$

This formula combined with (3.1) implies that

Letting $\varepsilon \downarrow 0$ we have

$$
E_{z}^{\pi}\left[\tilde{u}_{m}\left(Z_{t}^{\pi}\right)\right] \leqq \tilde{u}_{m}(z)+\varepsilon .
$$

$$
E_{z}^{\pi}\left[\tilde{u}_{m}\left(Z_{t}^{\pi}\right)\right] \leqq \tilde{u}_{m}(z)
$$

Next we shall show that for each $(x, s) \in Z$ and any $\pi \in I$,

$$
\underset{t \downarrow 0}{\liminf } \tilde{u}_{m}\left(Z_{s+t}^{\pi}\right) \geqq \tilde{u}_{m}(x, s) \quad P_{(x, s)}^{\pi}-\text { a.s. . }
$$

It follows by the excessiveness of $u^{*}$ that for each $(x, s) \in Z$, each $0 \leqq t \leqq t_{0}$ and any 
$\pi \in I$,

$$
\begin{aligned}
u_{m}(\pi)(x, s+t) & =E_{(x, s+t)}^{\pi}\left[u^{*}\left(Z_{\tau_{m}(s+t)}^{\pi}\right)\right] \\
& \geqq E_{(x, s+t)}^{\pi}\left[u^{*}\left(Z_{\tau_{m}}^{\pi}\left(s+t_{0}\right)\right)\right] .
\end{aligned}
$$

From Lemma 4.2 in [5], we get

$$
\begin{aligned}
\liminf _{t \downarrow 0} u_{m}(\pi)(x, s+t) & \geqq \liminf _{t \downarrow 0} E_{(x, s+t)}^{\pi}\left[u^{*}\left(Z_{\tau_{m}\left(s+t_{0}\right)}^{\pi}\right)\right] \\
& =E_{(x, s)}^{\pi}\left[u^{*}\left(Z_{\tau_{m}\left(s+t_{0}\right)}^{\pi}\right) .\right.
\end{aligned}
$$

Since $\tau_{m}\left(s+t_{0}\right) \downarrow \tau_{m}(s)$ as $t_{0} \downarrow 0$, by the right continuity of $u^{*}\left(Z_{t}^{\pi}\right)$ and Fatou's Lemma we have

$$
\begin{aligned}
\liminf _{t \downarrow 0} u_{m}(\pi)(x, s+t) & \geqq \liminf _{t_{0} \downarrow 0} E_{(x, s)}^{\pi}\left[u^{*}\left(Z_{\tau_{m}\left(s+t_{0}\right)}^{\pi}\right)\right] \\
& \geqq E_{(x, s)}^{\pi}\left[\liminf _{t_{0} \downarrow 0} u^{*}\left(Z_{\tau_{m}\left(s+t_{0}\right)}^{\pi}\right)\right] \\
& =E_{(x, s)}^{\pi}\left[u^{*}\left(Z_{\tau_{m}(s)}^{\pi}\right)\right] \\
& =u_{m}(\pi)(x, s) .
\end{aligned}
$$

Hence it follows from Lemma 4.3 in [5] that for any $\pi \in \Pi$ and each $(x, s) \in Z$,

$$
\liminf _{t \downarrow 0} \sup _{\tilde{\pi} \in \Pi} u_{m}(\tilde{\pi})\left(Z_{s+t}^{\pi}\right) \geqq \sup _{\tilde{\pi} \in \Pi} u_{m}(\tilde{\pi})(x, s) \quad P_{(x, s)}^{\pi}-\text { a.s., }
$$

that is,

$$
\underset{t \downarrow 0}{\liminf } \tilde{u}_{m}\left(Z_{s+t}^{\pi}\right) \geqq \tilde{u}_{m}(x, s) \quad P_{(x, s)}^{\pi}-\text { a.s. . }
$$

Finally from (3.2) and (3.3) $\tilde{u}_{m}$ is excessive.

LEMMA 3.2. For each $m \geqq 1$, the following hold:

(i) $\tilde{u}_{m}$ is a majorant of $g$.

(ii) $u^{*}(z)=\sup _{\pi \in \Pi} E_{z}^{\pi}\left[u^{*}\left(Z_{\sigma_{m}}^{\pi}\right)\right]=\tilde{u}_{m}(z)$ for all $z \in Z$.

ProOF. (i) Let

$$
c=\sup _{z \in Z}\left[g(z)-\tilde{u}_{m}(z)\right] .
$$

We have two cases : $c \leqq 0$ and $c>0$. In the first case, obviously $\tilde{u}_{m}(z) \geqq g(z)$ for all $z \in Z$.

Now suppose $c>0$. According to Lemma 3.1, $\tilde{u}_{m}$ is excessive, hence so is $c+\tilde{u}_{m}$. Also it is obvious that $c+\tilde{u}_{m}$ is a majorant of $g$. Since $u^{*}$ is the smallest excessive majorant of $g$, we get

$$
c+\tilde{u}_{m}(z) \geqq u^{*}(z) \quad \text { for } \quad z \in Z .
$$

We take $0<\alpha<\min \left(c, \frac{1}{m}\right)$. Then there is a point $z_{0}=\left(x_{0}, s_{0}\right) \in Z$ such that

$$
g\left(z_{0}\right)-\tilde{u}_{m}\left(z_{0}\right)>c-\alpha .
$$

Combining (3.4) and (3.5) leads us to

$$
0 \leqq u *\left(z_{0}\right)-g\left(z_{0}\right) \leqq c+\tilde{u}_{m}\left(z_{0}\right)-g\left(z_{0}\right)<\alpha<\frac{1}{m},
$$


that is,

$$
u^{*}\left(z_{0}\right)<g\left(z_{0}\right)+\frac{1}{m} .
$$

Thus the point $z_{0} \in \hat{\Gamma}_{m}^{*}$, where $\hat{\Gamma}_{m}^{*}$ is the set of all $(x, s) \in \Gamma_{m}^{*}$ for which there exists a $h_{0}>0$ depending on $(x, s)$ such that $(x, s+h) \in \Gamma_{m}^{*}$ for all $h \in\left[0, h_{0}\right)$. Hence it follows that for any $\pi \in \Pi \tau_{m}\left(s_{0}\right)=s_{0} P_{z_{0}}^{\pi}-$ a.s., and

$$
\tilde{u}_{m}\left(z_{0}\right)=\sup _{\pi \in \Pi} E_{z_{j}}^{\pi}\left[u^{*}\left(Z_{\tau_{m}}^{\pi}\right)\right]=u^{*}\left(z_{0}\right) \geqq g\left(z_{0}\right),
$$

which together with (3.5) yields the inequality $\alpha>c$, which contradicts the assertion $0<\alpha<\min \left(c, \frac{1}{m}\right)$. This completes the proof of (i).

(ii) By (i) and Lemma 3.1, $\tilde{u}_{m}$ is an excessive majorant of $g$. Since $u^{*}$ is the smallest excessive majorant of $g$, we have

$$
u^{*}(z) \leqq \tilde{u}_{m}(z) \quad \text { for } \quad z \in Z .
$$

On the other hand, it follows from the excessiveness of $u^{*}$ and $\tau_{m} \geqq \sigma_{m}$ that for any $\pi \in \Pi$,

hence

$$
u^{*}(z) \geqq E_{2}^{\pi}\left[u^{*}\left(Z_{\sigma_{m}}^{\pi}\right)\right] \geqq E_{2}^{\pi}\left[u^{*}\left(Z_{\tau_{m}}^{\pi}\right)\right],
$$

$$
u^{*}(z) \geqq \sup _{\pi \subseteq I} E_{z}^{\pi}\left[u^{*}\left(Z_{\sigma_{m}}^{\pi}\right)\right] \geqq \tilde{u}_{m}(z) \quad \text { for } \quad z \in Z .
$$

Therefore, from (3.6) and (3.7) we immediately obtain the desired equality

$$
u^{*}(z)=\sup _{\pi \in \Pi} E_{z}^{\pi}\left[u^{*}\left(Z_{\boldsymbol{\sigma}_{m}}^{\pi}\right)\right]=\tilde{u}_{m}(z) \quad \text { for } \quad z \in Z .
$$

For each $x \in S$, let $\frac{d^{+}}{d s} f(x, \cdot)$ denote the right-hand derivative of $f(x, \cdot)$.

Then we have

LEMMA 3.3. Suppose that:

(i) $\frac{d^{+}}{d s} u^{*}$ exists and is bounded.

(ii) For each $x \in S, \frac{d^{+}}{d s} u^{*}(x, \cdot)$ is right continuous on $R^{+}$.

Then it holds that for each $(x, s) \notin \Gamma^{*}, \sup _{\pi \in \mathbb{I}} \AA^{\pi} u^{*}(x, s)$ is right continuous at $s$.

Proof. From Lemma 5.1 in [5] we have that $u^{*} \in \mathscr{D}(\mathcal{A})$ and that for any $\pi \in I I$ and each $(x, s) \in Z$,

$$
\begin{array}{r}
\mathcal{A}^{\pi} u^{*}(x, s)=\frac{d^{+}}{d s} u^{*}(x, s)+\lambda(x, s, \pi(x, s))\left[\int_{S} u^{*}(y, s) Q(d y \mid x, s, \pi(x, s))\right. \\
\left.-u^{*}(x, s)\right] .
\end{array}
$$

Hence by the given conditions and the properties of $\pi, \lambda$ and $Q$, it follows that for any $\pi \in \Pi \mathcal{A}^{\pi} u^{*}(x, \cdot)$ is right continuous on $R^{+}$for fixed $x \in S$.

Let $(x, s) \notin \Gamma^{*}$ be arbitrary. From Assumption (B), it holds that for any $\varepsilon>0$ there exists a policy $\pi_{\varepsilon} \in \Pi$ such that 


$$
0 \leqq \sup _{\pi \in \Pi} A^{\pi} u^{*}(z)-\mathcal{A}^{\pi} \varepsilon u^{*}(z)<\varepsilon / 3
$$

for all $z \notin \Gamma^{*}$. Also by the right continuity of $A^{\pi} \varepsilon u^{*}(x, s)$ at $s$, there exists a $h_{0}>0$ such that

$$
\left|\mathcal{A}^{\pi_{\varepsilon}} u^{*}(x, s+h)-\mathcal{A}^{\pi_{\varepsilon}} u^{*}(x, s)\right|<\varepsilon / 3
$$

and $(x, s+h) \oplus \Gamma^{*}$ for all $h \in\left[0, h_{0}\right)$. Combining (3.9) and (3.10) we obtain

$$
\left|\sup _{\pi \in I I} A^{\pi} u^{*}(x, s+h)-\sup _{\pi \in I I} A^{*} u^{*}(x, s)\right|<\varepsilon \quad \text { for } h \in\left[0, h_{0}\right),
$$

which completes the proof.

We fix $h_{0}$ so that $0<h_{0}<\infty$ and define a stopping time by the following:

$$
\mu=\min \left[h_{0}, \nu\left(Z_{s}^{\pi}\right)\right] \quad \text { for } \pi \in \Pi \text { and } s \geqq 0 .
$$

LEMma 3.4. For each $(x, s) \in Z$, we have $\inf _{\pi \in \Pi} E_{(x, s)}^{\pi}[\mu]>0$.

Proof. Let $(x, s) \in Z$ be arbitrary and let

$$
G^{\pi}(x, s ; h)=P_{(x, s)}^{\pi}[\nu(x, s) \leqq h], \quad h \geqq 0 .
$$

Then for any $\pi \in \Pi$ we get

$$
\begin{aligned}
E_{(x, s)}^{\pi}[\mu] & =\left(1-G^{\pi}\left(x, s ; h_{0}\right)\right) \cdot h_{0}+\int_{0}^{h_{0}} w G^{\pi}(x, s ; d w) \\
& \geqq \exp \left\{-\int_{0}^{h_{0}} \lambda\left(x, s+s^{\prime}, \pi\left(x, s+s^{\prime}\right)\right) d s^{\prime}\right\} \cdot h_{0} \\
& \geqq e^{-M h_{0}} h_{0},
\end{aligned}
$$

since $0<\lambda(z, a)<M$ for all $(z, a) \in Z \times A$. Hence we have

$$
\inf _{\pi \in \Pi I} E_{(x, s)}^{\pi}[\mu] \geqq e^{-M h_{0}} \cdot h_{0}>0 \text {. }
$$

\section{The main result}

In this section we shall prove the main result of the paper, that is, the optimal return satisfies the optimality equation, by using the fundamental lemmas obtained in Section 3.

THEOREM 4.1. Suppose that:

(i) $\frac{d^{+}}{d s} u^{*}$ exists and is bounded.

(ii) For each $x \in S, \frac{d^{+}}{d s} u^{*}(x, \cdot)$ is right continuous on $R^{+}$.

Then $u^{*}$ satisfies the following equation:

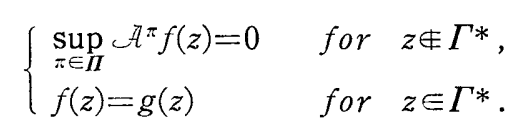

Proof. We shall first show that

$$
\sup _{\pi \in I I} \mathcal{A}^{\pi} u^{*}(z) \geqq 0 \quad \text { for } \quad z \in \Gamma^{*} .
$$


Suppose for some $\left(x_{0}, s_{0}\right) \notin \Gamma^{*}$,

$$
\sup _{\pi \in \boldsymbol{I I}} A^{\pi} u^{*}\left(x_{0}, s_{0}\right)<0 .
$$

Then there exists a $\delta>0$ satisfying

$$
\sup _{\pi \in \Pi} A^{\pi} u^{*}\left(x_{0}, s_{0}\right)+\delta<0 .
$$

Take $\varepsilon>0$ satisfying

$$
\sup _{\pi \in I I} \AA^{\pi} u^{*}\left(x_{0}, s_{0}\right)+\varepsilon+\delta<0 .
$$

From Lemma 3.3 it follows that there exists $h_{1}>0$ such that

$$
\sup _{\pi \in \Pi} A^{\pi} u^{*}\left(x_{0}, s_{0}+h\right)<\sup _{\pi \in \Pi} A^{\pi} u^{*}\left(x_{0}, s_{0}\right)+\varepsilon
$$

for all $h \in\left[0, h_{1}\right)$. From (4.1) and (4.2) we get

$$
\sup _{\pi \in \boldsymbol{I I}} \mathcal{A}^{\pi} u^{*}\left(x_{0}, s_{0}+h\right)+\delta<0 \quad \text { for } \quad h \in\left[0, h_{1}\right) \text {. }
$$

Since $\Gamma^{*}=\bigcap_{m=1}^{\infty} \Gamma_{m}^{*}$ and $\Gamma_{m}^{*} \supset \Gamma_{n}^{*}, n \geqq m$, it is obvious that there exist $h_{2}>0$ and $m_{0} \geqq 1$ such that $\left(x_{0}, s_{0}+h\right) \notin \Gamma_{m}^{*}$ for all $m \geqq m_{0}$ and all $h \in\left[0, h_{2}\right)$. Let $h_{0}=\min \left(h_{1}, h_{2}\right), \mu=$ $\operatorname{mim}\left[h_{0}, \nu\left(Z_{s_{0}}^{\pi}\right)\right]$ and let $\xi=s_{0}+\mu$. Then it holds from (4.3) that for any $\pi \in \Pi$,

hence

$$
\mathcal{A}^{\pi} u^{*}\left(Z_{t}^{\pi}\right)+\delta<0 \quad \text { for } \quad t \in\left[s_{0}, \xi\right) \quad P_{\left(r_{0}, s_{0}\right)}^{\pi}-\text { a.s. , }
$$

$$
E_{\left(x_{0}, s_{0}\right)}^{\pi}\left[\int_{s_{0}}^{\tilde{\xi}}\left(\mathcal{A}^{\pi} u^{*}\left(Z_{t}^{\pi}\right)+\delta\right) d t\right] \leqq 0
$$

By Lemma 5.4 in [5], we get

$$
u^{*}\left(x_{0}, s_{0}\right) \geqq E_{\left(x_{0}, s_{0}\right)}^{\pi}\left[u *\left(Z_{\xi}^{\pi}\right)\right]+\delta E_{\left(x_{0}, s_{0}\right)}^{\pi}[\mu] .
$$

Since $\xi \leqq \sigma_{m}<\infty$ for all $m \geqq m_{0}\left(P_{\left(x_{0}, 8_{0}\right)}^{\pi}-\right.$ a. s. $)$ and $u^{*}$ is excessive, we have

$$
E_{\left(x_{0}, s_{0}\right)}^{\pi}\left[u^{*}\left(Z_{\xi}^{\pi}\right)\right] \geqq E_{\left(x_{0}, s_{0}\right)}^{\pi}\left[u^{*}\left(Z_{\sigma_{m}}^{\pi}\right)\right]
$$

for any $\pi \in \Pi$ and each $m \geqq m_{0}$. This formula combined with (4.4) implies that

$$
\begin{aligned}
u^{*}\left(x_{0}, s_{0}\right) & \geqq E_{\left(x_{0}, s_{0}\right)}^{\pi}\left[u^{*}\left(Z_{\sigma_{m}}^{\pi}\right)\right]+\delta E_{\left(x_{0}, s_{0}\right)}^{\pi}[\mu] \\
& \geqq E_{\left(x_{0}, s_{0}\right)}^{\pi}\left[u^{*}\left(Z_{\sigma_{m}}^{\pi}\right)\right]+\delta \inf _{\pi \in \Pi} E_{\left(x_{0}, s_{0}\right)}^{\pi}[\mu]
\end{aligned}
$$

for any $\pi \in I I$ and each $m \geqq m_{0}$. Taking the supremum for $\pi \in \Pi$, we get

$$
u^{*}\left(x_{0}, s_{0}\right) \geqq \sup _{\pi \in \boldsymbol{I I}} E_{\left(x_{0}, s_{0}\right)}^{\pi}\left[u^{*}\left(Z_{\sigma_{m}}^{\pi}\right)\right]+\delta \inf _{\pi \in \Pi I} E_{\left(x_{0}, s_{0}\right)}^{\pi}[\mu] .
$$

Lemma 3.2 implies that

$$
u^{*}(z)=\sup _{\pi \in I I} E_{z}^{\pi}\left[u^{*}\left(Z_{\sigma_{m}}^{\pi}\right)\right]
$$

for all $m \geqq 1$ and all $z \in Z$. From (4.5) and (4.6) we obtain

$$
u^{*}\left(x_{0}, s_{0}\right) \geqq u^{*}\left(x_{0}, s_{0}\right)+\delta \inf _{\pi \in \Pi} E_{\left(x_{0}, s_{0}\right)}^{\pi}[\mu] .
$$


Since $\inf _{\pi \in I} E_{\left(x_{0}, s_{0}:\right.}^{\pi}[\mu]>0$ by Lemma 3.4,

$$
u^{*}\left(x_{0}, s_{0}\right)>u^{*}\left(x_{0}, s_{0}\right),
$$

which is a contradiction. So we get

$$
\sup _{\pi \in I I} \mathcal{A}^{\pi} u^{*}(z) \geqq 0 \quad \text { for } \quad z \notin \Gamma^{*} .
$$

Let $(x, s) \notin I^{*}$ and $\pi \in \Pi$ be arbitrary. We take sufficient small $h>0$ so that $(x, s+h) \notin \Gamma^{*}$, and define $\mu=\min \left[h, \nu\left(Z_{s}^{\pi}\right)\right]$ and $\xi=s+\mu$. Then by the excessiveness of $\imath^{*}$ we have

$$
\begin{aligned}
u^{*}(x, s) \geqq & E_{(,, s)}^{\pi}\left[u^{*}\left(Z_{\xi}^{\pi}\right)\right] \\
\geqq & \left(1-G^{\pi}(x, s ; h)\right) u^{*}(x, s+h) \\
& +\int_{0}^{h} \int_{s} G^{\pi}(x, s ; d w) Q(d y \mid x, s+w, \pi(x, s+w)) u^{*}(y, s+w) d w .
\end{aligned}
$$

From the properties of $\pi, \lambda$ and $Q$ it follows that for positive $h$ small enough,

$$
\begin{aligned}
& \frac{1}{h}\left\{u^{*}(x, s)-u^{*}(x, s+h)\right\} \\
& \geqq \frac{1}{h} \int_{0}^{h} \lambda^{\pi}(x, s+h) \exp \left(-\int_{0}^{w} \lambda^{\pi}\left(x, s+s^{\prime}\right) d s^{\prime}\right) \\
& \quad \cdot\left[\int_{s} u^{*}(y, s+w) Q^{\pi}(d y \mid x, s)-u^{*}(x, s+h)\right] d w,
\end{aligned}
$$

where

and

$$
\lambda^{\pi}(x, w)=\lambda(x, w, \pi(x, s)) \quad \text { for } \quad w \in[s, s+h),
$$

$$
Q^{n}(\cdot \mid x, s)=Q(\cdot \mid x, s, \pi(x, s)) .
$$

Letting $h \downarrow 0$ we have

$$
-\frac{d^{+}}{d s} u^{*}(x, s) \geqq \lambda^{\pi}(x, s)\left[\int_{s} u^{*}(y, s) Q^{*}(d y \mid x, s)-u^{*}(x, s)\right],
$$

which yields from (3.8) that

$$
\mathcal{A}^{\pi} u^{*}(x, s) \leqq 0
$$

Since the above inequality holds for any $\pi \in \Pi$ and every $(x, s) \notin \Gamma^{*}$, we have

$$
\sup _{\pi \in I I} \mathcal{A}^{\pi} u^{*}(z) \leqq 0 \quad \text { for } \quad z \notin \Gamma^{*} .
$$

Combining (4.7) and (4.8) we obtain

$$
\sup _{\pi \in \Pi} \mathcal{A}^{\pi} u^{*}(z)=0 \quad \text { for } \quad z \notin \Gamma^{*} .
$$

This completes the proof. 


\section{Acknowledgment}

The author would like to thank Professor N. Furukawa for his helpful advice and encouragement during the preparation of this paper.

\section{References}

[1] Blumevtal, R. M. and Getoor, R. K., Markov Processes and Potential Theory, Academic Press, New York, (1968).

[2] Doshi, B. T., Continuous time control of Markov processes on an arbitrary state space: discounted rewards, Ann. Statist. 4 (1976), 1219-1235.

[3] Fukukawa, N., Functional equations and Markov potential theory in stopped decision processes, Mem. Fac. Sci., Kyushu Univ., Ser. A, 29 (1975), 329-347.

[4] Kakcmasc, P., Continuously discounted Markov decision model with countable state and action spaces, Ann. Math. Statist., 42 (1971), 919-926.

[5] Oнtscio, Y., Controlled Markov jump processes associated with a choice of stopping rules, Mem. Fac. Sci., Kyushu Univ., Ser. A, (to appear).

[6] Shiryayev, A.N. Statistical sequential analysis, Transl. Math. Monograph., Amer. Math. Soc., (1973). 